\title{
Spin Dependent Conductance of a Quantum Dot Side attached to Topological Superconductors as a Probe of Majorana Fermion States
}

\author{
D. KRYChOWski ${ }^{a}, *$ S. Lipiński ${ }^{a}$ And G. Cuniberti ${ }^{b}$ \\ ${ }^{a}$ Institute of Molecular Physics, Polish Academy of Sciences, Smoluchowskiego 17, 60-179 Poznań, Poland \\ ${ }^{b}$ Institute for Materials Science and Max Bergmann Center of Biomaterials, TU Dresden, 01062 Dresden, Germany

\begin{abstract}
Spin-polarized transport through a quantum dot side attached to a topological superconductor and coupled to a pair of normal leads is discussed in Coulomb and Kondo regimes. For discussion of Coulomb range equation of motion method with extended Hubbard I approximation is used and Kondo regime is analyzed by KotliarRuckenstein slave boson approach. Apart from the occurrence of zero bias anomaly the presence of Majorana states reflects also in splitting of Coulomb lines. In the region of Coulomb borders the spin dependent negative differential conductance is observed. Due to the low energy scale of Kondo effect this probe allows for detection of Majorana states even for extremely weak coupling with topological wire. In this range no signatures of Majorana states appear in Coulomb blockade dominated transport.
\end{abstract}

DOI: 10.12693/APhysPolA.133.552

PACS/topics: 72.10.Fk, 73.63.Kv, 74.45.+c, 85.35.Gv

\section{Introduction}

Majorana fermions and Majorana bound states (MBSs) have attracted considerable interest in recent years due to their fundamental exotic properties e.g. self-hermicity and associated with this peculiarity nonAbelian statistics [1]. These features make Majorana states potential candidates for the use in fault-tolerant topological quantum computation [2]. MBSs are predicted to exist at the ends of a semiconductor nanowire with strong spin-orbit coupling placed in external magnetic field and brought into proximity of s-wave superconductor [3]. Also several other propositions for the realizations of $1 \mathrm{~d}$ topological superconductor (TS) have been reported e.g. carbon nanotubes with broken chiral symmetry and curvature induced spin-orbit coupling [4]. Various proposals have been made to detect the Majorana states using different hybrid structures based on quantum dots $[5,6]$. In this report we discuss signatures of Majorana states in transport through quantum dot coupled to normal leads and to a single or a pair of TS wires. Both Coulomb blockade range and Kondo regime are discussed. Due to the helical properties of TS wire its Majorana end-state hybridizes with only one of the dot spin orientations $[1,7]$, and thus in addition to conductance also polarization of conductance gives information on Majorana states. Present report complements the earlier studies on this topic by detailed analysis of spin polarization. We present the possibility of control of spin transport and we find the spin negative differential conductance, the phenomenon of wide array of po-

*corresponding author; e-mail: krychowski@ifmpan.poznan.pl tential applications e.g. in spin dependent amplifiers or switching circuits.

\section{Model and formalism}

T-shape system of Majorana bound state coupled to the quantum dot is presented in Fig. 1a (single side attached TS) and on the inset of Fig. 2a (a pair of TSs). The total Hamiltonian is $\mathcal{H}=\mathcal{H}_{0}+\mathcal{H}_{M B S}+\mathcal{H}_{D M} . \mathcal{H}_{0}$ is the Anderson hamiltonian and it describes the dot and the leads:

$$
\begin{aligned}
\mathcal{H}_{0} & =\sum_{\sigma} E_{d} n_{\sigma}+\mathcal{U} n_{\uparrow} n_{\downarrow}+\sum_{k \alpha=L, R \sigma} \varepsilon_{k \alpha \sigma} n_{k \alpha \sigma} \\
& +\sum_{k \alpha \sigma}\left(V c_{k \alpha \sigma}^{\dagger} d_{\sigma}+\text { H.c. }\right)
\end{aligned}
$$

where $E_{d}$ is QD level and $\varepsilon_{k \alpha \sigma}$ are energies of conduction electrons. The term parameterized by $\mathcal{U}$ describes Coulomb interaction and $V$ represents hopping between the dot and normal leads. $\mathcal{H}_{M B S}=\sum_{l} \mathrm{i} \delta_{l} \gamma_{1}^{l} \gamma_{2}^{l}$ describes coupling between the two Majorana states $\gamma_{1}^{l}, \gamma_{2}^{l}$ in the $l$ th TS wire $(l=1,2)$. Coupling between Majorana modes can be neglected in the case when TS wire is much longer than the coherence length $(L \gg \xi)$. In the opposite limit (dirty topological superconductors) $\delta \neq 0\left(\delta \sim e^{-L / \xi}\right)$. The Majorana operators can be expressed by fermionic operators: $\gamma_{1}^{l}=\left(f_{l}+f_{l}^{\dagger}\right) / \sqrt{2}, \gamma_{2}^{l}=\mathrm{i}\left(f_{l}-f_{l}^{\dagger}\right) / \sqrt{2}$. Due to helical properties of TS wire Majorana states are spin polarized [7]. The dot-Majorana coupling term is $\mathcal{H}_{D M}=t_{1} \gamma_{1}^{1}\left(-d_{\uparrow}+d_{\uparrow}^{\dagger}\right)+\mathrm{i} t_{2} \gamma_{2}^{2}\left(d_{\downarrow}+d_{\downarrow}^{\dagger}\right)$, where we assumed configuration in which spin polarizations of Majorana states $\gamma_{1}^{1}$ and $\gamma_{2}^{2}$ are opposite and thus electrons of one spin direction, say up, are coupled with MBS from the lower wire and spin down electrons with Majorana state of the upper wire. To find QD retarded Green's functions $G^{R}(t)=\left\langle\left\langle d_{\sigma}(t) ; d_{\sigma}^{\dagger}(0)\right\rangle\right\rangle$ in Coulomb 
blockade regime we use extended Hubbard I approximation. In Hubbard I approach two-particle Green's function $\left\langle\left\langle d_{\uparrow} n_{\downarrow} ; d_{\uparrow}^{\dagger}\right\rangle\right\rangle$ are decoupled as follows $\left\langle n_{\downarrow}\right\rangle\left\langle\left\langle d_{\uparrow} ; d_{\uparrow}^{\dagger}\right\rangle\right\rangle$, and in the extension we use they are treated exactly. Kondo regime is discussed within finite $\mathcal{U}$ slave boson a)
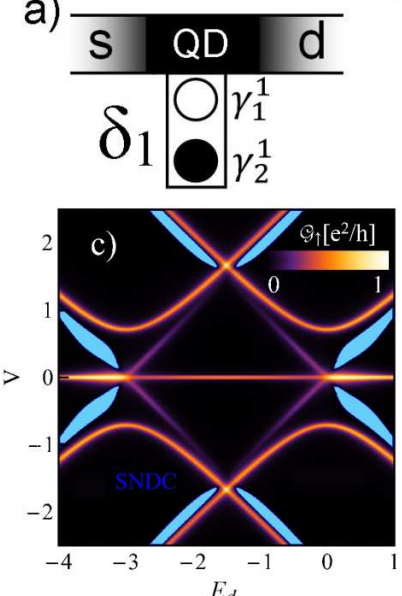

$E_{d}$

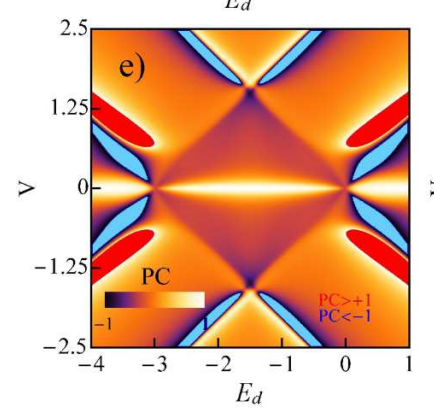

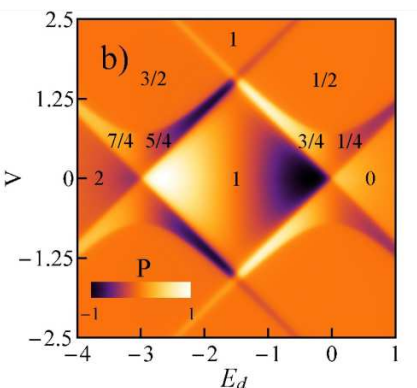
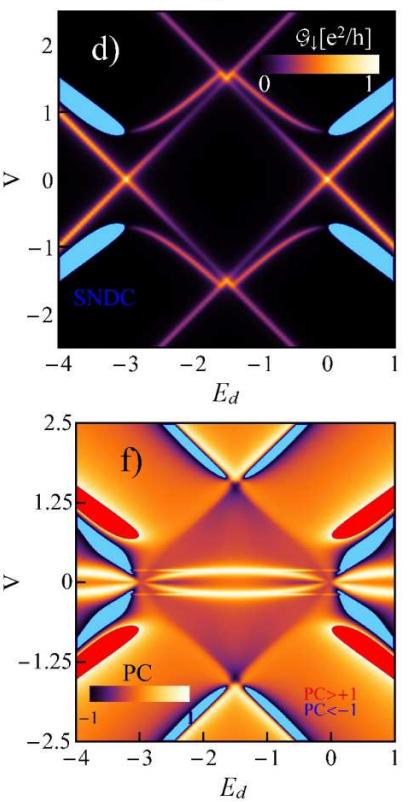

Fig. 1. a) Scheme of a quantum dot coupled to topological superconducting wire in T-shape geometry. White and black circles symbolize two Majorana states of opposite spin polarizations. Coulomb blockade regime: b) Spin accumulation map with occupation numbers on it. c) Conductance map for spin up orientation $\mathcal{G}^{\uparrow}\left(E_{d}, V\right)$ (blue areas denote the SNDC regions for this spin orientation. d) $\mathcal{G}^{\downarrow}\left(E_{d}, V\right)$ with marked SNDC regions for spin down orientation. e,f ) Plots of polarizations of conductance for $\delta=0(\mathrm{e})$ and $\delta=0.2(\mathrm{f})$. Red and blue regions correspond to $P C>+1$ and $P C<-1$ respectively $(\mathcal{U}=3, \Gamma=0.05, t=0.5$, the energy unit is defined in terms of the bandwidths $2 D=100$ ).

approach (K-R) [8], which maps the problem into the effective noninteracting particles picture with renormalized dot energy, hoppings to the leads and coupling to Majorana state. Since the only term not discussed so far in $\mathrm{K}-\mathrm{R}$ formalism is $\mathcal{H}_{D M}$, we give here its $\mathrm{SB}$ representation $\mathcal{H}_{D M}^{S B}=t_{1} \gamma_{1}^{1}\left(z_{\uparrow}^{\dagger} f_{\uparrow}^{\dagger}-z_{\uparrow} f_{\uparrow}\right)+\mathrm{i} t_{2} \gamma_{2}^{2}\left(z_{\downarrow}^{\dagger} f_{\downarrow}^{\dagger}+z_{\downarrow} f_{\downarrow}\right)$, where $z_{\sigma}=\left(e^{\dagger} p_{\sigma}+p_{\bar{\sigma}}^{\dagger} d\right) / \sqrt{\left\langle Q_{\sigma}\right\rangle\left(1-\left\langle Q_{\sigma}\right\rangle\right)}$ is the renormalization factor, with SB operator $e$ acting as projec- tor onto empty state, $p_{\sigma}$ onto single occupied state and $d$ onto doubly occupied state. $Q_{\sigma}=p_{\sigma}^{\dagger} p_{\sigma}+d^{\dagger} d$ is $\mathrm{SB}$ representation of the occupation number operator. Current flowing through the dot is expressed by $\mathcal{I}_{\sigma}=$ $(2 e / h) \int_{-\infty}^{+\infty}\left(f_{L}-f_{R}\right) T_{\sigma}(E) \mathrm{d} E$, where $f_{L(R)}$ are the Fermidistribution functions in the left and right electrodes and transmission $T_{\sigma}=-\Gamma_{\sigma} \Im\left[G_{\sigma, \sigma}^{R}\right] . \Gamma_{\sigma}=2 \pi V^{2} \varrho_{\sigma}\left(E_{F}\right)$, and $\varrho$ is DOS of the leads. The differential conductances are given by $\mathcal{G}_{s}=\mathrm{d} \mathcal{I}_{s} / \mathrm{d} V$ and the spin polarization of conductance is defined by $P C=\left(\mathcal{G}_{\uparrow}-\mathcal{G}_{\downarrow}\right) / \mathcal{G}$.
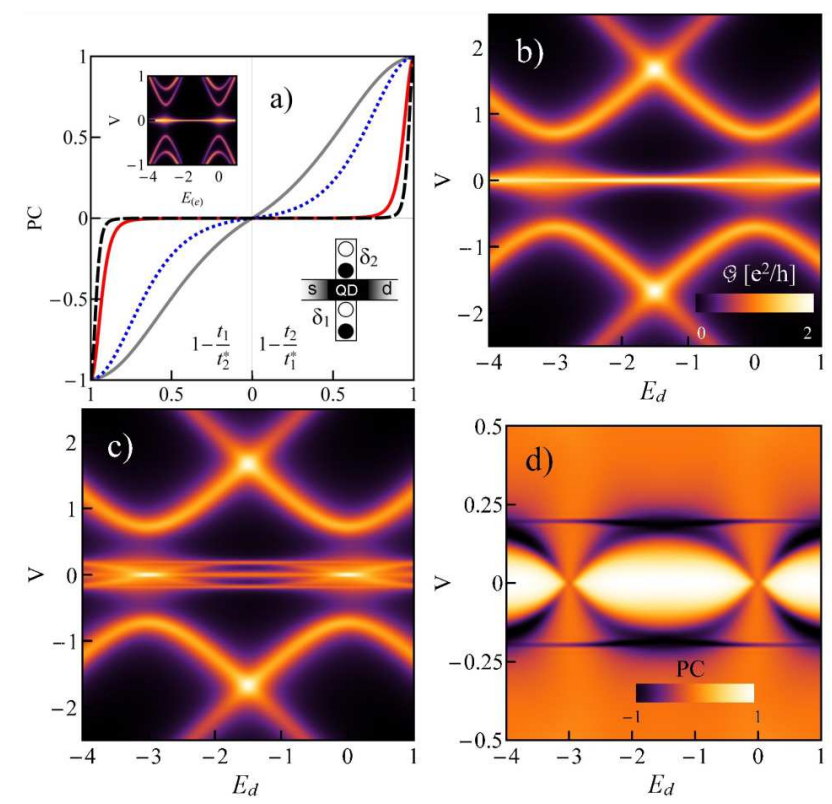

Fig. 2. a) Polarizations of conductance for $E_{d}=-\mathcal{U} / 2$ plotted vs. $t_{2}$ for several fixed values of $t_{1}=t_{1}^{*}$ (right axis) or vs. $t_{1}$ for fixed values of $t_{2}=t_{2}^{*}$ (left axis), $t_{1(2)}^{*}=0.005$ (solid gray line), $t_{1(2)}^{*}=0.1$ (dotted blue), $t_{1(2)}^{*}=0.5$ (solid red) and $t_{1(2)}^{*}=1$ (dashed black). Lower inset presents a scheme of quantum dot coupled to two TS wires and upper inset shows total conductance map for $t_{1}=0.5$ and $t_{2}=0.25(\Gamma=0.05)$. b,c) Differential conductance for $\delta_{1}=\delta_{2}=0$ (b) and $\delta_{1}=0, \delta_{2}=0.2(\mathrm{c})$. d) Polarization of conductance in the low bias region for $\delta_{1}=0, \delta_{2}=0.2(\Gamma=0.25$, $\left.t_{1}=t_{1}=0.5\right)$.

\section{Results}

First we discuss the impact of single Majorana state on Coulomb blockade considering QD with only one side attached TS wire $\left(t_{2}=0\right)$ (inset of Fig. 1a). Figs. 1c, $\mathrm{d}$ show the spin resolved differential conductance maps. Zero bias anomaly (ZBA) with conductance reaching $(1 / 2)\left(e^{2} / h\right)$ is only observed for spin-up channel. Another signature of Majorana-dot coupling is the observed splitting of Coulomb lines. Apart from typical Coulomb blockade diamonds also additional lines with nonlinear bias and gate voltage dependencies appear. The former lines are dominated by spin down electrons and the latter 
by tunneling of spin up carriers. Whereas spin up contribution is directly modified by Majorana-dot coupling, spin down is influenced only indirectly by the change of occupation. The splitting between ordinary Coulomb blockade point and excited lines at two degeneracies points $\left(E_{d}=0, E_{d}=-\mathcal{U}\right)$ is of order of $t$. Interesting observation is the occurrence of spin dependent negative differential conductance $\mathrm{d} \mathcal{I}_{\sigma} / \mathrm{d} V<0$ (SNDC) occurring for both spin orientations (blue regions at Figs. 1c, d). SNDC occurs on Coulomb blockade straight lines for spin up and on the curved Coulomb lines for spin down. To visualize this effect more clearly we present also spin polarization maps, where in the regions of SNDC, according to the definition of polarization of conductance, $P C>+1$ occurs for spin up NDC (red regions on Figs. 1d, e) and $P C<-1$ for spin down (blue regions). SNDC increases
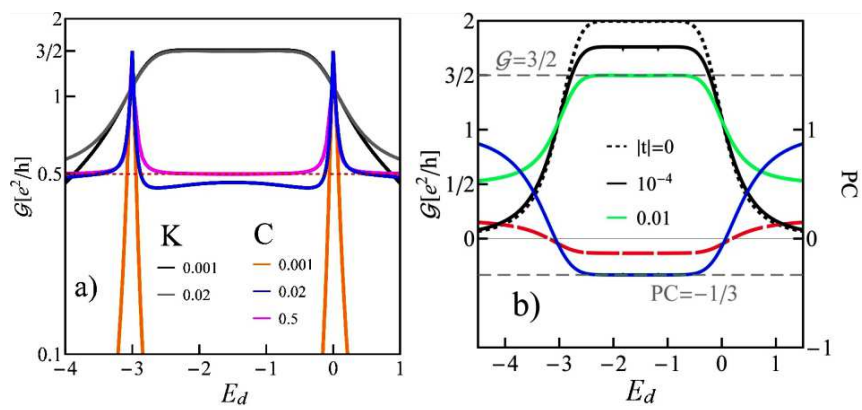

Fig. 3. a) Comparison of conductance in the Kondo $(\mathrm{K})$ and Coulomb blockade regimes $(\mathrm{C})$ for different values of coupling of the dot with TS wire t1 $(\mathrm{t} 2=0)$. b) Conductances for the $\mathrm{SU}(2)$ Kondo (dotted black line, $t_{1}=0$ ), for SU(2) Majorana-Kondo effect (solid green, $\mathcal{G}=(3 / 2))$ and for intermediate case (black line). The solid blue and dashed red lines represent the polarization of conductance for $t_{1}=10^{-4}$ and $t_{1}=0.01(\mathcal{U}=3$, $\Gamma=0.05)$.

with weakening of the coupling to the normal electrodes. Fig. If illustrates the effect of finite overlap between Majorana fermions from the opposite ends of the wire $\left(\delta_{1} \neq 0\right)$. ZBA resonance splits, but still remains fully spin polarized. Splitting is proportional to $\delta$. Fig. 1b presents the map of spin accumulation $\left(P=N_{\downarrow}-N_{\uparrow}\right)$ with marked occupation numbers. Note that in contrast to the unperturbed Coulomb blockade $(t=0)$, for the system coupled to MBS $(t \neq 0)$ the additional intermediate occupations occur $(N=1 / 4,3 / 4,5 / 4,7 / 4)$ in the region between Majorana split Coulomb lines. In this range also spin accumulation changes the sign with decrease of $E_{d}$. Fig. 2 illustrates the impact of two Majorana modes of opposite spin polarizations. Such a case can be realized with two side attached TS wires touching the dot with the opposite ends (Fig. 2a). For $\delta_{1}=\delta_{2}=0$ and symmetric coupling $\left(t_{1}=t_{2}\right)$ conductance of ZBA is doubled in comparison to the case of coupling with single wire $\left(G=\left(e^{2} / h\right)\right)$ and is unpolarized, because in tunneling equally engaged are carriers of both spin orientations. For asymmetric coupling linear conductance becomes spin polarized. Changing the tunneling amplitudes $\left(t_{1} / t_{2} \neq 1\right)$ one can modify the magnitude and the sign of polarization and these dependencies are sensitive to the strength of hopping parameters (Fig.2a). When Majorana modes from both wires are directly coupled to the dot typical Coulomb diamonds disappear and only nonlinear conductance lines are visible (inset of Fig. 2b). Finite coupling between two Majorana states in the wire leads to the earlier mentioned splitting of ZBA (Fig. 2c). If $\delta_{1} \neq \delta_{2}$ two pairs of finite bias lines appear characterized by opposite spin polarizations (not presented). Fig. $2 \mathrm{~d}$ illustrates the special case $\left(\delta_{1}=0\right.$ and $\left.\delta_{2}=0.2\right)$, where both zero and finite bias lines coexist and the corresponding polarizations of conductance are opposite. Changing the bias voltage one can switch between the reversed polarizations (Fig. 2d). Fig. 3 compares impact of $\mathrm{ZBA}$ on transport in the Coulomb range and Kondo regime and the main message is that due to the low energy scale of Kondo fluctuations only analysis of transport in this limit can be used in the case of weak coupling of the dot to TS wire. As it is seen from Fig. 3a for $t=0.001$ Coulomb blockade is undisturbed by the presence of MBS, contrary to Kondo case, where even for this small coupling distinct reduction of Kondo conductance is observed. Fig 3b shows the evolution of Majorana perturbed Kondo conductance with the increase of $t$ and the spin polarization for $t \neq 0$. The unitary conductance reaches $(3 / 2)\left(e^{2} / h\right)[5]$ for $N=1$ and $(1 / 2)\left(e^{2} / h\right)$ for $N=0,2$. For $\mathrm{SU}(2)$ Majorana-Kondo effect, the polarization of conductance is negative for positively polarized Majorana state $\left(\gamma_{1}\right)(P C=-1 / 3)$, what is in contrast to the Coulomb range, where $P C=1$.

\section{Acknowledgments}

This project was supported by the Polish National Science Centre from the funds awarded through the decision No. DEC-2013/10/M/ST3/00488.

\section{References}

[1] M. Leijnse, K. Flensberg, Phys. Rev. B 86, 104511 (2012).

[2] S. Das Sarma, M. Freedman, C. Nayak, NPJ Quant. Inform. 1, 15001 (2015).

[3] V. Mourik, K. Zuo, S.M. Vrolov, S.R. Plissard, E.P.A.M. Bakkers, L.P. Kouwenhoven, Science 336, 1003 (2012).

[4] J.D. Sau, S. Tewari, Phys. Rev. B 88, 054503 (2013).

[5] M. Lee, J.-S. Lim, H. Khim, R. López, Phys. Rev. B 87, 241402(R) (2012).

[6] P. Stefański, Acta Phys. Pol. A 127, 198 (2015).

[7] D. Sticlet, C. Bena, P. Simon, Phys. Rev. Lett. 108, 096802 (2012).

[8] G. Kotliar, A. E. Ruckenstein, Phys. Rev. Lett. 57, 1362 (1986). 